Saudi Journal of Oral and Dental Research

Abbreviated Key Title: Saudi J Oral Dent Res ISSN 2518-1300 (Print) |ISSN 2518-1297 (Online) Scholars Middle East Publishers, Dubai, United Arab Emirates Journal homepage: http://scholarsmepub.com/sjodr/

\title{
Comparative Study to Determine the Efficacy of Chemi-Luminescent Method (Vizilite) and Toludine Blue Staining as a Screening Test in Oral Pre-Malignant Lesions
}

\author{
Dr. Vikram Khanna ${ }^{1 *}$, Dr Ranjitkumar Patil ${ }^{2}$, Dr Anurag Tripathi ${ }^{3}$, Dr Vandana $\mathrm{Singh}^{4}$ \\ ${ }^{1}$ Associate Professor Department of Oral Medicine \& Radiology Faculty of Dental Sciences, KGMU, Lucknow, India \\ ${ }^{2}$ Professor \& Head Department of Oral Medicine \& Radiology Faculty of Dental Sciences, KGMU, Lucknow, India \\ ${ }^{3}$ Additional Professor Department of Oral Medicine \& Radiology Faculty of Dental Sciences, KGMU, Lucknow, India \\ ${ }^{4}$ Associate Professor Department of Oral Medicine \& Radiology Faculty of Dental Sciences, KGMU, Lucknow, India
}

\begin{abstract}
DOI: $10.36348 /$ sjodr.2019.v04i12.011
| Received: 06.06.2019 | Accepted: 24.06.2019 | Published: 25.12.2019
\end{abstract}

*Corresponding author: Dr. Vikram Khanna

\section{Abstract}

Objectives: The study was done to evaluate efficacy of chemiluminescent method (vizilite) as a screening test in detecting the malignant changes in potentially malignant lesions of oral cavity. Study also compares sensitivity and specificity of toludiene blue with chemiluminescent method. Materials \& methods: 30 patients clinically diagnosed as potentially malignant lesions of oral cavity were recruited. After obtaining consent form, all patients were subjected chemiluminescent method and toludiene blue. All patients were then compared with histopathological results and statistically analyzed. Results: After histopathological results, Sensitivity and specificity of vizilite was found to be $66.6 \%$ and $71.4 \%$ respectively and that of toluidine blue was $77 \%$ and $87 \%$. Conclusion: Although vizilite accentuated the visibility of the lesion but overall detection rate in patients was not significantly improved. Qualities like easy to perform, less time consuming and instant results concur with the properties of an ideal screening test but diagnostic propensity is questionable.

Keywords: Vizilite, Chemiluminescent method, toludiene blue, pre-cancerous lesions.

Copyright @ 2019: This is an open-access article distributed under the terms of the Creative Commons Attribution license which permits unrestricted use, distribution, and reproduction in any medium for non-commercial use (NonCommercial, or CC-BY-NC) provided the original author and source are credited.

\section{INTRODUCTION}

Squamous cell carcinoma accounts for the most common cancer affecting the oral cavity and continues to be enigmatic to the oral physician and oncologists. Unfortunately, the prognosis of this deadly entity relies on the time of patient presentation. Until now, the only method the dentist had in looking for signs of oral cancer was visual. By the time the dentist could see signs of oral cancer in the mouth, it was often in an advanced stage of development. Despite improved surgical approaches, reconstruction techniques and advances in radiation and medical oncology, the single most effective route of improving the long term out come of oral cancer is early diagnosis. One such tool which can play an important role in limiting this disease in juvenile stage is the Screening tests. Oral cancer is an ideal cancer to identify early by screening. Various types of screening test kits based on the differential properties of dysplastic oral cells are available in market.
Vizilite is a chemiluminescent based visualization method which helps oral physician to detect the lesion in much earlier stage. Theoretically, the special Vizilite light will be absorbed by normal tissue, but will cause abnormal, pre-cancerous, or cancerous lesions to appear white. Another tool which could be accepted indicator of oral cancers is toluidine blue. It is a metachromatic dye which is consistent with loss of chromosomal genetic information.

In present study we evaluated the effectivity of vizilite (Chemiluminescent method) as a screening test in detecting the malignant changes, based on histological report and compared with toluidine blue staining, in pre-cancerous lesion.

\section{MATERIALS AND METHODS}

The present study was conducted in department of Oral Medicine \& Radiology. The study was observational study to test the efficacy of chemiluminescent method (vizilite) and toludiene blue staining in pre-cancerous lesions. 30 patients clinically 
diagnosed as pre-cancerous lesion were selected and after obtaining the consent from the patients they were randomly subjected to the study. Vizilite, in accordance with the instructions given by the company (Zila pharmaceuticals) was used to visualize the clinically diagnosed pre-cancerous lesion and the color of reflected light was noted. Subsequently, area was marked with indelible pencil. The marked area was subjected to the toluidine blue staining.

To assess the efficacy of both the procedures scalpel biopsy was done from the area showing maximum amount of the dye uptake.

\section{RESULTS AND OBSERVATIONS}

Out of 30 leukoplakic patients observed with Vizilite, 18 showed no change in light reflections, 12 showed increased brightness and sharpness of the white patch (table 1). The dye retention was negative in the 17 patients who showed no visualization with Vizilite and 1 patient showed dye retention. Out of 12 patients who showed enhanced light 7 patients showed dye retention. Rest 5 patients did not exhibit any change in dye application (table 2).

The scalpel biopsy report confirmed no dysplastic cells in the 15 of 17 patients who neither showed white light reflection nor increased dye uptake and 2 cases showed mild dysplasia. Dysplastic cells were evident in the 7 cases who exhibited both increased light reflection and dye retention. Rest of the cases demonstrated no signs of cellular atypia.

Sensitivity and specificity of vizilite was found to be $66.6 \%$ and $71.4 \%$ respectively and that of toluidine blue was $77 \%$ and $87 \%$.

Table-1: Table showing results of vizilite

\begin{tabular}{|l|l|l|}
\hline & Disease \\
\hline Test (vizilite) & Positive & Negative \\
\hline Positive & 6 & 6 \\
\hline Negative & 3 & 15 \\
\hline
\end{tabular}

Table-2: Table showing results of Toludiene blue

\begin{tabular}{|l|l|l|}
\hline & \multicolumn{2}{|l|}{ Disease } \\
\hline Test (toluidine blue) & Positive & Negative \\
\hline Positive & 7 & 1 \\
\hline Negative & 2 & 20 \\
\hline
\end{tabular}

\section{DISCUSSION}

According to the recent data, more than 300,000 people worldwide diagnosed with oral cancer every year. In India, incidence [1] of squamous cell carcinoma is 30 per 100,000 . Most common site involved in oral cavity is Bucco-gingival sulcus, alveolar mucosa, lip, tongue and palate, in contrast to the involvement of lip as most frequent in other countries. This may attribute to the higher rate of consumption of smokeless tobacco among the people of
India. Other causes are smoking tobacco, alcohol, poor diet, Epstein Barr virus and loss of heterozygosity near genome 9p21. Early detection of the cancer might be the solution in curbing the menace and therefore, might reduce the mortality and morbidity. Studies confirm that survival does correlate with stage, making early diagnosis and treatment optimal for this disease. The above described study is a genuine attempt to equate both the tests against the gold standard i.e. scalpel biopsy to evaluate their potency in detection of mucosal abnormality.

According to the figures given by the company, Vizilite has got the sensitivity of $100 \%$ in detecting white lesions but in our study, however in our study it was found to be $66.6 \%$. This might be due to the fact that we included only pre-cancerous lesion in study and proves non- specificity of vizilite in detecting the dysplasia. Regarding toluidine blue, sensitivity and specificity mentioned in the literature[2] is $77 \%$ and $67 \%$ and present study disclosed $77 \%$ and $87 \%$.Similarly et al.[3] assessed the adjunctive value of a chemiluminescent light source and application of pharmaceutical grade toluidine blue and observed that chemiluminescent exam improves the brightness and/or sharpness of margin in $61.8 \%$ of identified lesions. Biopsied lesions with toluidine blue stain retention reduced the false positive rate by $55.26 \%$ while maintaining a $100 \%$ negative predictive value (NPV). He inferred that chemiluminescent can be used as an adjunct in the practice.

Contrastingly, $\quad$ Oh ES and Laskin DM[4] investigated the efficacy of the individual components of Vizilite for improved visualization of early mucosal lesions and found that chemiluminescent light produced reflections made visualization more difficult, and thus the ViziLite ${ }^{\circledR}$ System was not beneficial.

On comparing the sensitivity and specificity, toluidine blue is more reliable than the visualization method. Gandolfo et al. ${ }^{[5]}$ also assessed the histological features of in vivo toluidine blue (TB) uptake in potentially malignant oral lesions and showed that toluidine blue had more nuclear uptake in malignant lesions than benign lesions. Gupta A et al.[6] evaluated the usefulness of toluidine blue and brush biopsy in precancerous oral lesions and squamous cell carcinoma and confirmed that it can be used as screening test in pre-cancerous lesions.

The number of false positive for chemiluminescent method is more in lesions such as traumatic ulcer; lichen planus can show the change in the intensity of reflected light. Similarly, Farah CS, McCullough MJ[7] carried out a pilot case control study on the efficacy of acetic acid wash and chemiluminescent illumination (ViziLite) in the visualization of oral mucosal white lesions. They concluded that ViziLite illumination does not 
discriminate between keratotic, inflammatory, malignant or potentially malignant oral mucosal white lesions.

Positive predicative value of vizilite and toluidine blue is $50 \%$ and $87 \%$ respectively which shows the faithfulness of toluidine blue towards dysplasticity of the lesion. On evaluating the negative predictive value, both the test more or less, exhibits same values i.e. $83 \%$ and $90 \%$.

Combining the results of both tests increases the overall performance and makes it easier for the oral physician in arriving at the diagnosis of malignancy in its incipient form.

\section{CONCLUSION}

Considering the fact that number of oral cancer patients is on rise, it is need of hour to have a test which could detect the mucosal changes in its puerile stage. Although vizilite accentuated the visibility of the lesion but overall detection rate in patients was not significantly improved. In fact, vizilite has showed the even in those lesions which were clinically not diagnosed as potential malignant. No doubt, qualities like easy to perform, less time consuming and instant results concur with the properties of an ideal screening test but diagnostic propensity is questionable.

\section{REFERENCES}

1. Sanderson, R.J., Ironside, J.A.D. (2002). Squamous cell carcinomas of the head and neck: commentary
Head and Neck carcinomas in developing countries. BMJ; 325:822-827

2. Onofre, M.A., Sposto, M.R., Navarro, C.M. (2001). Reliability of toluidine blue application in the detection of oral epithelial dysplasia and in situ and invasive squamous cell carcinomas. Oral Surg Oral Med Oral Pathol Oral Radiol Endod. May; 91(5):535-40.

3. Epstein, J. B., Silverman Jr, S., Epstein, J. D., Lonky, S. A., \& Bride, M. A. (2008). Analysis of oral lesion biopsies identified and evaluated by visual examination, chemiluminescence and toluidine blue. Oral oncology, 44(6), 538-544.

4. Oh, E. S., \& Laskin, D. M. (2007). Efficacy of the ViziLite system in the identification of oral lesions. Journal of oral and maxillofacial surgery, 65(3), 424-426.

5. Gandolfo, S., Pentenero, M., Broccoletti, R., Pagano, M., Carrozzo, M., \& Scully, C. (2006). Toluidine blue uptake in potentially malignant oral lesions in vivo: clinical and histological assessment. Oral oncology, 42(1), 88-94.

6. Gupta, A., Singh, M., Ibrahim, R., \& Mehrotra, R. (2007). Utility of toluidine blue staining and brush biopsy in precancerous and cancerous oral lesions. Acta cytologica, 51(5), 788-794.

7. Farah, C. S., \& McCullough, M. J. (2007). A pilot case control study on the efficacy of acetic acid wash and chemiluminescent illumination (ViziLite ${ }^{\mathrm{TM}}$ ) in the visualisation of oral mucosal white lesions. Oral oncology, 43(8), 820-824. 